Original

\title{
Papel actual de la fotovaporización prostática con Láser Greenlight HPS. Aspectos técnicos y revisión de la literatura
}

\author{
Carlos Capitán Manjón, José M. de la Morena Gallego, Enrique de la Peña Zarzuelo, \\ Victoria Gómez dos Santos, Carlos Llorente Abarca
}

Servicio de Urología. Hospital Universitario Fundación Alcorcón, Madrid, España.

\begin{abstract}
Resumen
La fotovaporización prostática (FVP) con láser Greenlight HPS 120W es una de las modalidades para el tratamiento quirúrgico de la hiperplasia benigna de la próstata (HBP) más atractivas en la actualidad. Las características físicas propias de este láser le dotan de un excelente perfil de seguridad y eficacia, convirtiéndola en la técnica ideal para pacientes de alto riesgo quirúrgico o con anticoagulación/antiagregación activa. Además, la incuestionable disminución del tiempo de sondaje y de hospitalización con respecto a la resección transuretral de la próstata (RTUp), implica un postoperatorio inmediato mucho más cómodo para los pacientes.

Hemos realizado una búsqueda bibliográfica en Medline/Pubmed, presentando en esta revisión los resultados de los estudios más recientes, así como las actuales técnicas quirúrgicas. También hemos revisado las limitaciones y aspectos controvertidos que se atribuyen a esta técnica en función de lo publicado en la literatura.
\end{abstract}

Palabras clave: Hiperplasia benigna de próstata. Fotovaporización prostática. Láser Greenlight. Triborato de litio.

\section{Photoselective vaporization of the prostate with Laser Greenlight HPS: current role, technical aspects and review of the literature}

\section{Abstract}

Photoselective vaporization of the prostate with 120W HPS Greenlight laser is one of the most attractive modalities of surgical treatment for benign prostatic hyperplasia at present. The specific physical characteristics of this laser provides an excellent safety and effectiveness profile, making it the ideal technique for high-risk patients or patients ongoing oral anticoagulation. Moreover, the undeniable reduction of urethral catheterization time and hospital stay compared to transurethral resection of prostate (TURP) involves a much more comfortable postoperative period for patients.

We have conducted a Medline/Pubmed search, presenting in this review the outcomes and current surgical techniques described in recent studies. We have also reviewed the controversial aspects and limitations attributed to this technique.

Keywords: Benign prostatic hiperplasia. Prostate fotovaporización. Greenlight laser. Lithium triborato.

$\mathrm{L}$ a RTUp se considera el tratamiento quirúrgico de elección para los síntomas del tracto urinario inferior secundarios a HBP. Intentando disminuir las complicaciones asociadas a este procedimiento, pero manteniendo sus resultados a largo plazo, se han desarrollado distintas modalidades de tratamiento con distintos sistemas de creación de energía, la mayoría de ellos sin mucho éxito. Es el caso de la ablación transuretral con agujas (TUNA), microondas transuretrales (TUMT), coagulación intersticial por láser, láser de neodimio...Todas estas técnicas han demostrado un aceptable perfil de seguridad y eficacia a corto y medio plazo, pero el elevado porcentaje de pacientes con disconfort post- operatorio, así como unos malos resultados a largo plazo, ha hecho que la mayoría hayan caído en desuso.

Disponemos actualmente de una técnica mínimamente invasiva cuyos resultados parecen prometedores: la fotovaporización prostática (FVP) con láser Greenlight HPS 120W. Los primeros pasos con esta técnica fueron dados por Malek en la Clínica Mayo en $1998^{1}$ utilizando un láser de 60W (la mitad de potencia que los actuales). Desde entonces numerosos estudios ${ }^{2-4}$ han aportado datos que demuestran que se trata de una técnica con una tasa de complicaciones muy baja y unos excelentes resultados postoperatorios. Al mismo tiempo que 
hemos conocido estos estudios se han producido sustanciales mejoras en el sistema láser, principalmente un aumento de la potencia, lo que ha permitido disminuir significativamente el tiempo quirúrgico manteniendo el perfil de seguridad y eficacia.

Por otra parte, también se pueden encontrar objeciones a esta técnica. Una de ellas es el elevado coste económico, aunque no existen en la literatura estudios concluyentes al respecto, y los disponibles hasta ahora determinan que la FVP es menos cara que la RTUp ${ }^{5,6}$. Otro de los puntos débiles no cuestionables de la FVP es la ausencia de estudios prospectivos aleatorizados randomizados con seguimiento a largo plazo, que avalen los resultados hasta ahora publicados (a diferencia de su gran "competidor" que es la enucleación prostática con láser Holmium). Sin duda uno de los responsables de este hecho es el desarrollo de esta técnica en el ámbito de la medicina privada, al menos en nuestro medio.

Creemos que la FVP con láser HPS 120W es la modalidad de tratamiento más atractiva en la actualidad para el tratamiento de la HBP con indicación quirúrgica. En esta revisión presentamos los datos disponibles en la literatura que avalan dicha creencia.

\section{FÍSICA DEL LÁSER Y TÉCNICA QUIRÚRGICA}

El láser Greenligth HPS (High Performance System) emite la energía con una longitud de onda de 532nm, lo cual le coloca en el área visible verde del espectro electromagnético. Esta longitud de onda es absorbida de forma casi completa por cualquier tejido rico en oxihemoglobina (coeficiente de absorción 102/cm), como el tejido prostático ${ }^{7}$, siendo muy poco absorbida en el líquido de irrigación (coeficiente de absorción 104/cm). Ejerce su acción calentando el agua intracelular del tejido sobre el que se aplica, resultando en una vaporización inmediata del mismo (hecho que se constata durante la realización del procedimiento por la presencia de burbujas) al mismo tiempo que produce una coagulación inducida por calor de los vasos superficiales. Dos de sus principales características son: una profundidad de penetración de únicamente 1-2mm y una frecuencia de pulso quasi-continua para prevenir el efecto de "calentamiento acumulado", lo que se traduciría en una extensa necrosis coagulativa. Son estas características las que hacen que el láser produzca una fina capa de coagulación con pérdida hemática mínima ${ }^{8,9}$.
El láser de 80W, conocidos por mucho como KTP, genera su energía pasando un láser de Neodimium: yttrium aluminium garnet (Nd: Yag) con una longitud de onda de $1064 \mathrm{~nm}$ a través de un cristal de potasio-titanil-fosfato (KTP). Con esto se consigue doblar la frecuencia y dividir por 2 la longitud de onda. El nuevo láser HPS, mantiene la misma longitud de onda que su predecesor (532 $\mathrm{nm}$ ), pero utiliza un cristal de triborato de litio en vez del KTP. Con esto se consigue aumentar la potencia hasta $120 \mathrm{~W}$, aumentado a su vez la eficacia y la velocidad de la vaporización. Otras mejoras del nuevo sistema son un haz de luz más colimado, lo que permite trabajar a mayor distancia del tejido con el mismo rendimiento en la vaporización ${ }^{10}$, un sistema de refrigeración por aire en lugar del antiguo sistema por agua (que exigía la presencia de una toma de agua en quirófano) y la presencia de 2 pedales independientes, uno para vaporización (60-120W) y otro para coagulación (20-40W).

La energía láser es aplicada en el tejido mediante una fibra de disparo lateral de 700 micras, que se pasa por el canal de trabajo de un cistoscopio de flujo continuo entre 21-23F. Para una correcta fotovaporización prostática es necesario que el tejido y la fibra no estén en contacto directo, pero la distancia entre ambos no debe ser superior a 4-5mm ("near-contact technique"). Como líquido de irrigación se utiliza suero salino isotónico, lo que unido a la ausencia de sangrado hace que el riesgo de síndrome de reabsorción sea prácticamente nulo. También se acepta en la literatura la utilización de agua como irrigación, pero el riesgo de reabsorción es mayor ${ }^{11}$.

La finalidad del procedimiento es la creación de una cavidad prostática lo suficientemente amplia como para permitir una micción confortable. Es importante insistir en mantener una distancia fibra-tejido no mayor a $5 \mathrm{~mm}$ (una distancia menor se traduciría en un mayor riesgo de rotura de la fibra y una distancia mayor haría que el efecto predominante fuera la coagulación con el consiguiente aumento de la clínica irritativa). La formación de burbujas es signo de FVP efectiva, y típicamente, según nos acercamos a la cápsula prostática disminuyen el tamaño de dichas burbujas, por tratarse de un tejido más fibrótico y con menos hemoglobina. Se han descrito distintas técnicas de vaporización. En la técnica original descrita por 
Malek et al. ${ }^{12}$ la FVP comienza en el cuello de la vejiga y se dirige al veru montanum vaporizando de un lóbulo al otro en el sentido de las agujas del reloj. En caso de lóbulo medio prominente se recomienda comenzar por el mismo para garantizar suficiente flujo como para mantener una visión adecuada $^{13}$. La vaporización ha de ser muy cuidadosa en los lóbulos apicales para no causar daños en el esfínter externo ${ }^{14}$. Otra técnica descrita es la FVP en espiral: en este caso la vaporización comienza en el lóbulo medio, continúa por los lóbulos laterales y termina en el apex. ${ }^{15}$. La finalidad de esta técnica es vaporizar completamente un área de tejido antes de pasar a otra, pues el tejido previamente vaporizado se vuelve más denso y por lo tanto la eficacia de vaporización disminuye. Shandu et al. ${ }^{16}$ introduce una variación técnica para próstatas de gran tamaño descrita como vaporización-incisión y consistente en realizar primero una incisión en el lóbulo medio hasta el trígono, seguidas de 2 incisiones inmediatamente laterales al lóbulo medio y otras 2 en la parte alta de ambos lóbulos laterales. Queda asî la próstata dividida en porciones, que se vaporizando consecutivamente. No se recomienda esta técnica a cirujanos sin experiencia por el riego de dañar orificios ureterales ${ }^{7}$.

Recientemente, intentando estandarizar la técnica quirúrgica, el International GreenLight User Group (IGLU) ha propuesto un planteamiento quirúrgico por escalones, que es el utilizado por nuestro grupo, consistente en: cistoscopia previa, creación de canal de trabajo, vaporización de lóbulos laterales, lóbulos apicales y por último cuello/lóbulo medio $^{17}$. Recomendamos comenzar a $80 \mathrm{~W}$ hasta la creación de un canal de trabajo lo suficientemente amplio como para trabajar sin contacto directo con el tejido, lo que permite disminuir el riego de degradación de la fibra por el roce continúo con el tejido. También recomendamos trabajar con 80w cerca de los puntos de riesgo como son el cuello prostático, lóbulo medio y zona apical.

Las complicaciones más importantes que pueden surgir durante la fotovaporización son:

- Daño orifico ureteral, que puede ocurrir vaporizando lóbulos medios de gran tamaño, o cuando se trabaja en el cuello, pues la fibra dispara a $70^{\circ}$ y no a $90^{\circ}$

- Daño esfínter externo, trabajando cerca del veru montanum-apex.
- Hematuria. El principal enemigo de la FVP es el sangrado, no tanto por la intensidad (que normalmente es mínima) sino por la dificultad de visión que conlleva al trabajar con un cistoscopio de pequeño calibre. Para controlar el sangrado tenemos varias posibilidades: lo primero continuar vaporizando en otro punto pues la mayoría de las veces cede sin otra maniobra. También se puede utilizar la función de coagulación del sistema, aumentar la distancia fibra-tejido, o aumentar la velocidad de rotación. Sin embargo, en nuestra experiencia la mayoría de los sangrados se producen por movimientos bruscos del cistoscopio, por lo que la medida más eficaz es intentar no realizar este tipo de movimientos.

Al finalizar el procedimiento, se coloca sonda vesical (18-20F) que se mantiene al menos $12 \mathrm{~h}$, lo que permite que en algunos casos el paciente pueda ser dado de alta el mismo día de la intervención (procedimiento de hospital de día) ${ }^{18}$.

Una de las ventajas que se le atribuye a la FVP con láser HPS es la razonablemente corta curva de aprendizaje, al contrario que la enucleación prostática con láser de holmium (HoLEP). Rajbabu et al. ${ }^{19}$ considera que son necesarios entre 10 y 20 procedimientos con próstatas pequeñas $(<50 \mathrm{cc})$ para realizar una FVP correcta y con seguridad. BucherHayes $^{20}$ cree sin embargo que son necesarios tan sólo 5 procedimientos para próstatas pequeñas (<40cc) y 20 procedimientos para próstatas de mayor tamaño.

Basándonos en nuestra experiencia, creemos que es imprescindible diseñar un programa de formación a varios niveles antes de la implantación del procedimiento. En primer lugar; un período de formación básica, con la adquisición y conocimiento del equipo e instrumental tanto de los cirujanos como del personal de enfermería. En segundo lugar; un período de aplicación hospitalaria. Como en otras técnicas novedosas la estancia en centros de reconocido prestigio previo a la realización de los primeros procedimientos es necesaria, así como la invitación de urólogos con amplia experiencia a nuestro centro para colaborar en estos primeros casos. Estimamos que son necesarios al menos 1520 procedimientos con próstatas menores de 50cc, sin grandes lóbulos medios y sin anticoagulación/antiagregación activa para pasar a un escalón superior en dificultad. 


\section{¿SIGUE SIENDO LA RTU PROSTÁTICA EL "GOLD STANDARD"?}

Cualquier nueva técnica quirúrgica para el tratamiento de la HBP debe ser comparada con la RTUp, que ha demostrado sobradamente su eficacia y seguridad a lo largo del tiempo. La RTUp es la segunda cirugía más frecuente en el mundo Occidental $^{21}$, aunque su frecuencia ha ido disminuyendo en EEUU desde 350.000 procedimientos en 1986 a 200.000 en $1998^{22}$.

A pesar de su seguridad demostrada, no es un procedimiento exento de complicaciones como: hematuria con necesidad de transfusión (4\%), ITU (1$3 \%)$, síndrome de reabsorción (1-2\%), resondaje (6\%), eyaculación retrograda (70\%-100\%), disfunción eréctil (3-35\%), estenosis de uretra (3-4\%) o incontinencia $(0,5-3 \%)^{23,24}$. Tiene una tasa de mortalidad entre 0,2 $1,5 \%$ y una morbilidad en conjunto del $11,1 \%^{25,26}$ con un porcentaje de reintervención del 6-13\% ${ }^{27}$.

Por todas estas razones es necesario revisar nuestra percepción en cuanto a seguridad y eficacia de la RTUp, clarificando su actual papel en el tratamiento de la HBP, especialmente teniendo en cuenta las nuevas técnicas emergentes.

\section{EFICACIA Y SEGURIDAD}

En los últimos 10 años numerosos autores han presentado su experiencia en distintos trabajos ${ }^{1,3,4,12,28-32}$, todos ellos con el láser 80W. Casi todos ellos aportan unos excelentes resultados a corto plazo, con mejoras sustanciales en los parámetros de vaciamiento (IPSS y $\mathrm{Gmax}$ ). En la mayoría la sonda se retira antes de las $24 \mathrm{~h}$, con una estancia hospitalaria inferior a los 2 días (incluso en un estudio no se deja sonda la finalizar el procedimiento ${ }^{4}$ ). Las principales complicaciones en el postoperatorio inmediato reflejadas son hematuria leve (4-18\%), disuria-urgencia (7-30\%), ITUS (6\%) y RAO (1-5\%). Los datos con mayor variabilidad entre unos estudios y otros es el de la reducción del volumen prostático que varía entre el $37-53 \% \%^{30,32,36}$.

En un estudio muy interesante, Hamann et al. ${ }^{34}$ analiza los parámetros urodinámicos a un año de 135 pacientes sometidos a FVP. Todos ellos presentaban una mejora en el IPSS, así como una disminución de la presión detrusoriana y del volumen residual. Sólo 4 casos $(5,1 \%)$ se consideraron urodinámicamente obstruidos al año. Destacar que aproximadamente el $40 \%$ presentaron alguna molestia miccional en el postoperatorio inmediato.
Todos estos datos hacen que la FVP sea presentada como un procedimiento eficiente y muy bien tolerado, aunque existe escepticismo en los resultados a largo plazo. El único estudio que tiene un seguimiento largo ${ }^{1}$ mantiene unos resultados a 5 años muy buenos $(79 \%$ del los pacientes mantenían un $100 \%$ de la mejoría en el qmax). Sin embargo, sólo 14 de los 94 pacientes iníciales completaron el seguimiento y sólo 15 pacientes se trataron con el láser de 80w.

\section{FVP LÁSER vS RTUP}

Unos de los "puntos débiles" que se le atribuyen a la FVP es la falta de estudios randomizados prospectivos a largo plazo comparándose con la RTUp, como el que se está realizando en nuestro centro actualmente. Aún así, encontramos en la literatura 3 estudios prospectivos no randomizados y uno prospectivo randomizado comparando FVP con láser 80W y RTUp ${ }^{2,35-37}$.

Bachmann et al. ${ }^{35}$ comparan 64 pacientes sometidos a FVP con 37 intervenidos mediante RTUp y presentan sus resultados a 6 meses. La mejoría de Qmax e IPSS son similares en ambos grupos, pero la FVP reducía significativamente el tiempo de sondaje y de ingreso hospitalario y provocaba un descenso menor en las cifras de hemoglobina y sodio. Sin embargo, la disminución del volumen prostático era significativamente mayor con la RTUp.

Bouchier et al. ${ }^{2}$ presentan 120 pacientes aleatoriamente randomizados a FVP y RTUp con un seguimiento de 1 año. Al igual que Bachmann presentan unos resultados de eficacia muy similares en ambos grupos, con un claro descenso del tiempo de sonda y hospitalización a favor de la FVP. Un aspecto novedoso que aporta este estudio es que refiere una menor tasa de complicaciones en el grupo de la FVP, lo que implicaría una reducción de los costes del $22 \%$. Similares conclusiones se extraen del estudio de Tugcu et al. ${ }^{36}$.

En un reciente trabajo de Ruszat et al. ${ }^{37}$ analizan 396 pacientes con 2 años de seguimiento y categorizados por edades. Encuentran resultados significativamente superiores en la FVP en cuanto a sangrado intraoperatorio (3vs1 1\%), necesidad de transfusión (0vs5,5\%), perforación capsular $(0,4 \mathrm{vs} 6,3 \%)$ y retención urinaria postoperatoria $(0,4 v s 3,9 \%)$. Sin embargo, la mejoría de qmax fue mejor con la RTUp, aunque la mejoría de IPSS y volumen residual fue similar. La reducción de volumen prostático a los 12 
meses fue del $63 \%$ para la RTUp y del $44 \%$ para la FVP. No hubo diferencias estadísticamente significativas en el porcentaje de reintervenciones aunque fue mayor en el grupo de la FVP $(6,7 \% v s 3,9 \%)$.

No existe ningún trabajo randomizado prospectivo con láser HPS 120W.

Analizaremos a continuación los datos que se extraen de la literatura sobre aquellos aspectos controvertidos sobre la utilización del láser HPS 120W en determinadas circunstancias como próstatas de gran tamaño, pacientes de alto riesgo quirúrgico o anticoagulados y pacientes con sondaje de larga evolución.

\section{PRÓSTATAS GRANDES}

Una de las limitaciones que clásicamente se le han atribuido a la FVP ha sido el tratamiento de próstatas grandes (mayores de 70-80cc) debido al elevado tiempo quirúrgico que lleva el procedimiento, el mayor número de fibras necesarias y la elevada posibilidad de un segundo procedimiento. Los datos publicados hasta ahora son contradictorios.

Sandhu et al. ${ }^{38}$ trataron 64 pacientes con próstatas mayores de 60 cc con láser KTP 80W. La mediana de tamaño fue de 101 cc y la mediana de tiempo quirúrgico de 123 minutos. A pesar que el $28 \%$ de los pacientes eran portadores de sonda vesical más de 1 mes previo a la cirugía, en el 95\% de los casos se retiró antes de 23 horas de postoperatorias. La mejoría al año del $\mathrm{Qmax}$ fue de $7,9 \mathrm{ml} / \mathrm{sg}$ a $18,9 \mathrm{ml} / \mathrm{sg}$ y la disminución en la puntación IPSS fue de 18,4 a 6,7 .

Alivizatos et al. ${ }^{39,40}$ comparan en un estudio prospectivo randomizado adenomectomía y FVP con láser 80 W en 125 pacientes con próstatas de más de 80cc. Los datos han sido publicados con un tiempo de seguimiento de 12 y de 18 meses. Con la FVP el tiempo quirúrgico fue mayor (80 $\mathrm{min}$ vs $50 \mathrm{~min}$ ), pero el tiempo de sondaje vesical (24 vs $120 \mathrm{~h}$ ) y de hospitalización (48 vs 144h) fue claramente inferior. En cuanto a las complicaciones fueron similares en ambos grupos, salvo por una mayor tasa de transfusiones en el caso de la adenomectomía. No hubo diferencias estadísticamente significativas en la puntuación del IPSS, Qmax o volumen residual a los 18 meses. En lo que sí existen diferencias es en el volumen prostático medido después de la cirugía a favor del grupo de la adenomectomía.
Horasanli et al. ${ }^{41}$ en otro estudio prospectivo randomizado comparan FVP con láser KTP 80W y RTUp en 65 pacientes con próstatas por encima de los 70cc y nos muestran sus resultados a 6 meses de seguimiento. En este estudio, encuentran diferencias estadísticamente significativas a favor de la RTUp en la puntuación del IPSS (6,4 vs 13,1), Qmax (20,7 vs 13,3$)$, volumen residual $(22,9$ vs 78,9$)$ y porcentaje de reducción de volumen prostático. Malek $^{42}$ sugiere que los malos datos de éste se deben a que la experiencia de este grupo no era suficiente para llevar a cabo este tipo de intervenciones.

No existe, que sepamos, ningún estudio con el nuevo láser HPS de 120W tratando próstatas de los tamaños descritos.

\section{PACIENTES DE ALTO RIESGO gUIRÚRGICO}

Una de las claras ventajas de la FVP es la posibilidad de utilizarse en pacientes de alto riesgo quirúrgico con elevada comorbilidad, debido a la práctica ausencia de sangrado. La publicación más importante en este sentido la encontramos en un estudio de 2 centros en donde se evalúan 66 pacientes con una puntuación ASA (American Society of Anesthesiologists) de 3 o más ${ }^{32}$. No fueron necesarias transfusiones ni se detectaron síndromes de reabsorción. Un $11 \%$ de los pacientes tuvieron que ser resondados en el postoperatorio inmediato y sólo 1 precisó reintervención en el primer mes por persistencia de sintomatología obstructiva grave. Con un año de seguimiento encontraron una mejora del $222 \%$ de $\operatorname{Bmax}$ y una reducción de la puntuación del IPSS de 14 puntos.

$\mathrm{Fu}$ et al. $^{43}$ presentan resultados de seguridad similares en otro grupo de pacientes de alto riesgo. Sin embargo, algunos de los datos que presentan en su serie son cuanto menos sorprendentes, como es una media de tiempo de quirúrgico de sólo 25,6 minutos para una mediana de volumen prostático de $72,5 \mathrm{cc}$.

\section{PACIENTES CON ANTICOAGULACIÓN/ANTIAGREAGACIÓN}

En la práctica urológica habitual cada vez son más frecuentes los pacientes con anticoagulación o antiagregación activa que precisan cirugía prostática. La RTUp convencional no aporta un perfil de seguridad adecuado, con una tasa de transfu- 
sión en este tipo de pacientes de hasta el 30\%44. El cambio previo de la anticoagulación oral por heparinas de bajo peso no ha conseguido disminuir tanto como se esperaba las complicaciones en el postoperatorio, manteniendo una tasa de transfusiones de hasta el 20\%, con el consiguiente aumento del tiempo de hospitalización y sondaje vesical ${ }^{45}$.

Ruszat et al. ${ }^{46}$ presentan 116 pacientes que fueron intervenidos mediante FVP anticoagulación (warfarina) o antiagregación (aspirina o clopidrogel) activa y los compara con una serie de pacientes sometidos a FVP pero no anticoagulados. Ambos grupos presentaban similares datos de eficacia en cuanto a mejora de sintomatología y del flujo máximo. No se realizó ninguna transfusión en ninguno de los grupos, ni se detectaron complicaciones tromboembólicas graves. La única diferencia fue la necesidad de sueros lavadores durante más tiempo en el caso de los pacientes anticoagulados, con el consiguiente aumento del tiempo de sondaje. A los 2 años de seguimiento ambos grupos presentaban datos similares de mejoría.

\section{PACIENTES EN RETENCIÓN}

Tradicionalmente los pacientes sondados de larga duración por RAO se han asociado con un mayor número de complicaciones después de cirugía desobstructiva, así como con una mejoría más discreta en los parámetros de vaciamiento. En un estudio de Ruszat et al. ${ }^{47}$ compara 70 pacientes en RAO con 113 sin RAO todos ellos sometidos a FVP y con un seguimiento de 2 años, no encontrando diferencias estadísticamente significativas ni en complicaciones perioperatorias ni en la tasa de retención postoperatoria (12,9\%vs 10,6\%). Tampoco encontró diferencias estadísticamente significativas en puntuación del IPSS, $\mathrm{Q}$ Max o volumen residual. Sorprendentemente la media de estancia hospitalaria fue de 5,3 días en el primer grupo y 5,5 en el segundo, a pesar que la sonda se retira a los 1,7 días y 1,8 días respectivamente. Esto se puede explicar por las características especiales de los sistemas de salud de los países de los autores.

Un estudio más reciente de Hirst et al. ${ }^{48}$ compara pacientes en 2 grupos de pacientes en RAO tratados con FVP y RTUp. A 1 año de seguimiento los resultados de eficiencia son muy similares aunque a los 3 meses la puntuación del IPSS fue mejor en el brazo de de la RTUp.
En nuestra experiencia, la necesidad de sondaje después de FVP en pacientes sondados es alta si se retira la sonda entre $12-24$ h después de la cirugía por lo que dejamos la sonda al menos $48 \mathrm{~h}$ en este tipo de pacientes. No creemos que sea necesario dejar catéter suprapúbico para medir residuos, a diferencia de algunos grupos.

\section{CONCLUSIONES}

La FVP con láser Greenlight ofrece un excelente perfil de seguridad y eficacia, ofreciendo innegables ventajas tanto a pacientes como a Urólogos. Sus características físicas la convierten en una técnica ideal para pacientes de alto riesgo quirúrgico o con anticoagulación/antiagregación activa. Además, la incuestionable disminución del tiempo de sondaje y de hospitalización con respecto a la RTUp, implica un postoperatorio inmediato mucho más cómodo para los pacientes, así como una disminución en el gasto sanitario asociado a este procedimiento. La corta curva de aprendizaje en comparación con otros procedimientos, supone para los urólogos otra ventaja de gran valor.

Sin embargo, todavía no están disponibles en la literatura datos a largo plazo en estudios prospectivos que den mayor validez a los datos hasta ahora publicados. Además, no tenemos estudios concluyentes que valoren el coste de este tipo de tratamiento en relación con la RTUp, aunque los datos de los que disponemos consideran la FVP como una técnica con buena relación coste-efectividad. Tampoco queda claro si los resultados obtenidos en próstatas de menos de 70-80cc son extrapolables a próstatas de mayor tamaño.

Prácticamente no existe ningún estudio publicado con el nuevo sistema HPS 120W, que ha introducido mejoras en la efectividad de la vaporización y sobre todo en la velocidad de la misma, lo que ha permitido acortar claramente el tiempo quirúrgico.

\section{REFERENCIAS}

1. Malek R, Kuntzman R, Barrett D. Photoselective potassium-titanyl-phosphate laser vaporization of the benign obstructive prostate: observations on long term outcomes. J Urol 2005; 174:1344-1348.

2. Bouchier D, Anderson P, Van Appledon S, et al. KTP laser versus transureth ral resection:early results of a randomized trial. J Endourol 2006; 20:580 585 .

3. Bachmann A, Ruszat R, Wyler S, et al. Photoselective vaporization of the pros tate: the basal experience after 108 procedures. Eur Urol 2005;47:798-804.

4. Te A, Malloy TR, Stein BS, et al. Photoselective vaporization of the prostate for the treatment of benign prostatic hyperplasia: 12 months results from the first United States multicenter prospective study. J Urol 2004; 172:1404-1408.

5. Alivizatos G, Skolarikos A: Photoselective vaporization of the prostate. Review of cost implementation to BPH treatment. Prostate Cancer Prostatic Dis 2007, 10:15-20. 
6. Stovsky MD, Griffiths RI Duff SA .A Clinical Outcomes and Cost Analysis Comparing Photoselective Vaporization of the Prostate to Alternative Minimally Invasive Therapies and Transurethral Prostate Resection for the Treatment of Benign Prostatic Hyperplasia. J Urol 2006, 176: 1500-1506.

7. Te AE: The next generation in laser treatments and the role of the GreenLight High- Performance System Laser. Rev Urol 2006, 8:S24-S30.

8. Barber N, Muir G. High-power KTP laser prostatectomy: the new challenge to transurethral resection of the prostate. Curr Opin Urol 2004; 14:21-25.

9. Anson K. Could the latest generation potassium titanyl phosphate lasers be the ones to make transurethral resection of the prostate an operation of historical interest only? Curr Opin Urol 2004; 14:27-29.

10. Te AE: Current state of the art photoselective vaporization prostatectomy: laser therapy for benign prostatic hyperplasia. Prostate Cancer Prostatic Dis 2007,10:2-5.

11. Rajbabu K, Dudderidge T, Barber N, et al.: Evaluation of ideal irrigation fluid in "Greenlight" photoselective vaporization of the prostate. Prostate Cancer Prostatic Dis 2007, 10:101-103.

12. Malek RS, Kuntzmann RS, Barrett DM: High power potassium-titanylphosphate laser vaporization prostatectomy. J Urol 2000, 163:1730-1733.

13. Bachmann A, Ruszat R: The KTP-(greenlight-) laser-principles and experiences. Minim Invasive Ther Allied Technol 2007; 16:5-10.

14. Bachmann A, Ruszat R, Wyler S, et al.: Photoselective vaporization of the prostate: The Basel experience after 108 procedures. Eur Urol 2005 47:798-804.

15. Rajbabu K, Muir GH: GreenLight photoselective vaporization of prostate: a technical review. Prostate Cancer Prostatic Dis 2007, 10:6-9

16. Sandhu JS, Te AE: Photoselective vaporization of the prostate:the vaporization incision technique for large volume prostates [abstract]. J Urol 2005 , 173:366

17. Muir G, Sancha F, Bachmann A, et al. Techniques and training with GreenLight HPS 120-W laser therapy of the prostate: position paper. Eur Urol Supp 12008; 7:S370-S377.

18. Kaplan S: Expanding the role of photoselective vaporization of the prostate. Rev Urol 2006, 8:S3-S8.

19. Rajbabu K, Muir GH: GreenLight photoselective vaporization of prostate: a technical review. Prostate Cancer Prostatic Dis 2007, 10:6-9.

20. Bouchier-Hayes DM: Photoselective vaporization of the prostate-towards a new standard. Prostate Cancer Prostatic Dis 2007, 10:10-14.

21. Reich O, Gratzke C, Stief C. Techniques and long-term results of surgical procedures for BPH. Eur Urol 2006; 49:970-978.

22. Borth C, Beiko D, Nickel J. Impact of medical therapy on transurethral resection of the prostate: a decade of change. Urology 2001; 57:1082-1085.

23. Borboroglu P, Kane C, Ward J, et al. Immediate and postoperative complications of transurethral prostatectomy in the 1990s. J Urol 1999; 162:1307 1310.

24. Flanigan R, Reda D, Wasson J, et al. 5-year outcome of surgical resection and watchful waiting for men with moderately symptomatic benign prostatic hyperplasia: a Department of Veterans Affairs cooperative study. J Urol 1998; 160:12-16; discussion 16-17.

25. Horninger W, Unterlechner H, Strassr H, et al. Transurethral prostatectomy: mortality and morbidity. Prostate 1996; 28:195-200.

26. Reich O, Gratzke K, Backmann A, et al. Morbidity, mortality and early outcome of transurethral resection of the prostate: a prospective multicenter evaluation of 10654 patients. J Urol 2008; 180:246-249.

27. Roos N, Wennberg J, Malenka D, et al. Mortality and re-operation after open and transurethral resection of the prostate for benign prostatic hyperplasia. N Engl J Med 1989; 320:1120-1124.

28. Malek R, Kuntzmann R, Barrett D. KTP laser prostatectomy: long term experience. J Urol Suppl 2001; 165:369.

29. Sulser T, Reich O, Wyler S, et al. Photoselective KTP laser vaporization of the prostate: first experiences with 65 procedures. J Endourol 2004; 18:976-981.

30. Kumar S. Photoselective vaporization of the prostate: a volume reduction analysis in patients with lower urinary tract symptoms secondary to benign prostatic hyperplasia and carcinoma of the prostate. J Urol 2005; 173:511-513.

31. Volkan T, Ihsan T, Yilmaz O, et al. Short term outcomes of high power (80W) potassium-titanyl-phosphate laser vaporization of the prostate. Eur Urol 2005; 48:608-613.

32. Reich O, Bachmann A, Siebels M, et al. High power (80W) potassium titanylphosphate laser vaporization of the prostate in 66 high risk patients J Urol 2005; 173:158-160.
33. Sarica K, Alkan E, Luleci H, Tasci A. Photoselective vaporization of the enlarged prostate with KTP laser: long-term results in 240 patients. J Endourol 2005; 19:1199-1202.

34. Hamann M, Naumann C, Steif C, van der Horst C, Jünemann KP, Braun PM. Functional outcome following photoselective vaporisation of the prostate PVP): urodynamic findings within 12 months follow-up. Eur Urol 2008 54:902-910.

35. Bachman A, Schurch L, Ruzet R, et al. Photoselective vaporization (PVP) versus transurethral resection of the prostate (TURP): a prospective bi-center study of peri-operative morbidity and early functional outcome. Eur Urol 2005; 48:956-972.

36. Tuctu V, Tasci AI, Sain S et al Comparison of photoselective vaporization of the prostate and transurethral resection of the prostate: a prospective nonrandomized bicenter trial with 2-year follow-up. J Endourol 2008; 22:1519 1525.

37. Ruszat R, Wyler S, Seitz M, et al. Comparison of potassium-titanyl-phosphate laser vaporization of the prostate and transurethral resection of the prostate: update of a prospective nonrandomized two-centre study. BJU Int 2008.

38. Sandhu J, Ng C, Vanderbrink B, et al. High-power potassium-titanyl-phosphate photoselective laser vaporization of prostate for treatment of benign prostatic hyperplasia in men with large prostates. Urology 2004 64:1155-1159.

39. Alivizatos G, Skolarikos A, Chalikopoulos D, et al. Transurethral photoselective vaporization versus transvesical open enucleation for prostatic adenomas of more than $80 \mathrm{ml}: 12$-month results of a randomized prospective study. Eur Urol 2008; 54:427-437.

40. Skolarikos A, Alivizatos G et al. Eighteen-Month Results of a Randomized Prospective Study Comparing Transurethral Photoselective Vaporization with Transvesical Open Enucleation for Prostatic Adenomas Greater Than 80 cc. J. Endourol 2008; 22 :2333-2340

41. Horasanli K, Silay MS, Altay B, et al. Photoselective potassium titanyl phosphate (KTP) laser vaporization versus transurethral resection of the prostate for prostates larger than $70 \mathrm{ml}$ : a short-term prospective randomized trial. Urology 2008; 71:247-251.

42. Malek R. Photoselective potassium titanyl phosphate (KTP) laser vaporization of the prostate (PVP) vs transurethral resection of the prostate (TURP). Urology 2008; 72:718-719.

43. Fu WJ, Hong BF, Wang XX, et al.: Evaluation of greenlight photoselective vaporization of the prostate for the treatment of high-risk patients with benign prostatic hyperplasia. Asian J Androl 2006; 8:367-371.

44. Parr NJ, Loh CS, Desmond AD: Transurethral resection of the prostate and bladder tumor without withdrawal of warfarin therapy. Br J Urol 1989 64:623-625.

45. Dotan ZA, Mor Y, Leibovitch I, et al.: The efficacy and safety of perioperative low molecular weight heparin substitution in patients on chronic oral anticoagulant therapy undergoing transurethral prostatectomy for bladder outlet obstruction. J Urol 2003, 168:610-613.

46. Ruszat R, Wyler S, Forster T, et al.: Safety and effectiveness of photoselective vaporization of the prostate (PVP) in patients on ongoing oral anticoagulation. Eur Urol 2007, 51:1031-1041.

47. Ruszat R, Wyler S, Seifert HH, et al.: Photoselective vaporization of the prostate: subgroup analysis of men with refractory urinary retention. Eur Urol 2006, 50:1040-1049.

48. Hirst G, Edwards M, James W, Bose P: Comparison of TURP and photoselective vaporization of the prostate (PVP) in men presenting with painfu acute urinary retention[abstract]. Eur Urol 2007, 6:192.

Correspondencia autor: Dr. Carlos Capitán Manjón

Servicio de Urología

Hospital Universitario Fundación Alcorcón

Budapest, 1 - 28922 Alcorcon (Madrid)

Tel.: 916219400

E-mail autor: ccapitan@fhalcorcon.es

Información artículo: Original - HBP

Trabajo recibido: abril 2009

Trabajo aceptado: mayo 2009 